\title{
Medical Humanities in der Ausbildung
}

\section{Alexander Kiss ${ }^{a}$, Claudia Steinera, Alexander Honold ${ }^{b}$, Paul Grossman ${ }^{a}$, Franziska Gygax ${ }^{c}$, Ludwig Heuss $^{d}$, Wolf Langewitz ${ }^{a}$, Sylvie Schuster ${ }^{e}$ Claudia Kiessling ${ }^{f}$}

${ }^{a}$ Klinik Psychosomatik, Universitätsspital Basel; ${ }^{b}$ Deutsches Seminar, Universität Basel; ${ }^{c}$ Englisches Seminar, Universität Basel; ${ }^{\text {a }}$ Medizinische Klinik Spital

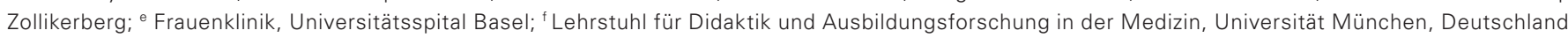

Medical Humanities, schon länger für viele Medizinstudierende in den USA und Kanada ein Pflichtteil ihres Curriculums, hat in letzter Zeit auch in der Schweiz Fuss gefasst. Dabei hat das Fach, wie durch die Schweizerischen Akademien der Wissenschaften dokumentiert, in den Medizincurricula der verschiedenen Universitäten einen unterschiedlichen Stellenwert [1].

Wie auch die Genfer Erfahrungen zeigen, sind vielen Medizinstudierenden die Inhalte von Medical Humanities und insbesondere die Auseinandersetzung mit den Perspektiven von Geisteswissenschaften und Kunst primär fremd und bedürfen eines spezifischen pädagogischen Ansatzes [2]. Für eine nachhaltige Verankerung solcher Inhalte ins Medizincurriculum ist es daher bedeutsam zu wissen, wie die Akzeptanz solcher auf den ersten Blick fachfremder Themen bei den Studierenden ist.

\section{Medical Humanities in Basel}

An der Medizinischen Fakultät der Universität Basel wurde Medical Humanities 1998 als eigenständiges Lehrangebot eingeführt, zuerst als freiwillige, später als verpflichtende Veranstaltungen, ergänzt durch fakultative Kurse (Tab. 1). Die Zusammenarbeit von Geisteswissenschaftlern, Künstlern und Medizinern für die Planung und Durchführung war von Anfang an entscheidend. Ein Leitgedanke war, dass immer auch ein Kliniker an der Veranstaltung beteiligt ist, um so einen möglichen Transfer von Medical Humanities in den späteren klinischen Alltag aufzuzeigen.

Tabelle 1: Medical Humanities in Basel.

\begin{tabular}{llll}
\hline Studienjahr & Veranstaltung & Obligat/fakultativ & Umfang \\
\hline Bachelor 1. Jahr & Film und Medizin & 2 von 3 obligat & $2 \times 4$ Stunden \\
\hline Bachelor 2. Jahr & Literatur und Medizin & 2 von 3 obligat & $2 \times 4$ Stunden \\
\hline Bachelor 3. Jahr & Medical Mindfulness & Fakultativ & 20 Stunden \\
& Transkulturelle Kompetenz & Fakultativ & 20 Stunden \\
\hline Master 1. Jahr & Reflective Writing & Obligat & \\
\hline
\end{tabular}

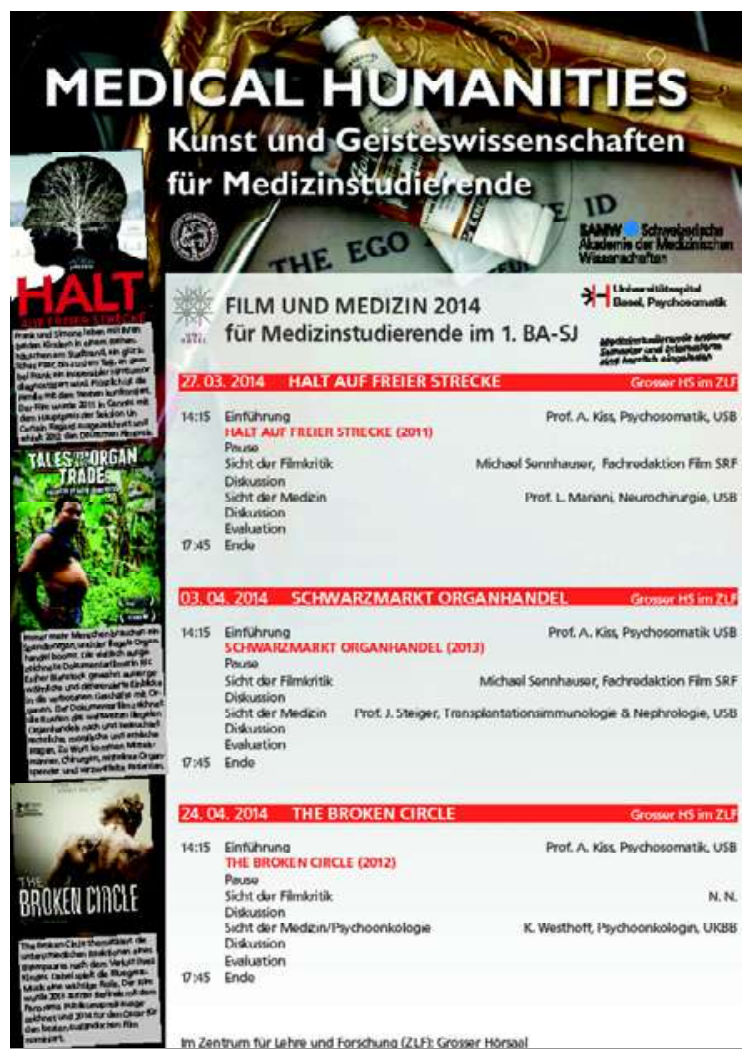

Abbildung 1: Ausschnitt aus der Programmankündigung «Film und Medizin».

Die Programmankündigung zu «Film und Medizin» (Abb. 1) zeigt exemplarisch den Ablauf der Veranstaltung. 2013 wurde von der Curriculumskommission die Besuchspflicht auf zwei von drei Veranstaltungen von jeweils «Film und Medizin» und «Literatur und Medizin» erhöht.

In Weiterentwicklung dieses Angebots findet seit 2011 Reflective Writing im Rahmen des Einzeltutoriats [3] statt. Die Studierenden arbeiten dabei während zwei Semestern einen Nachmittag in der Woche in einer Hausarztpraxis mit. Im Rahmen des Reflective Writing beschreiben und reflektieren sie schriftlich eine $\mathrm{Pa}$ tientenbegegnung (z.B. «Begegnung mit einem $\mathrm{Pa}$ tienten, für den Sie wenig oder keine Empathie emp- 


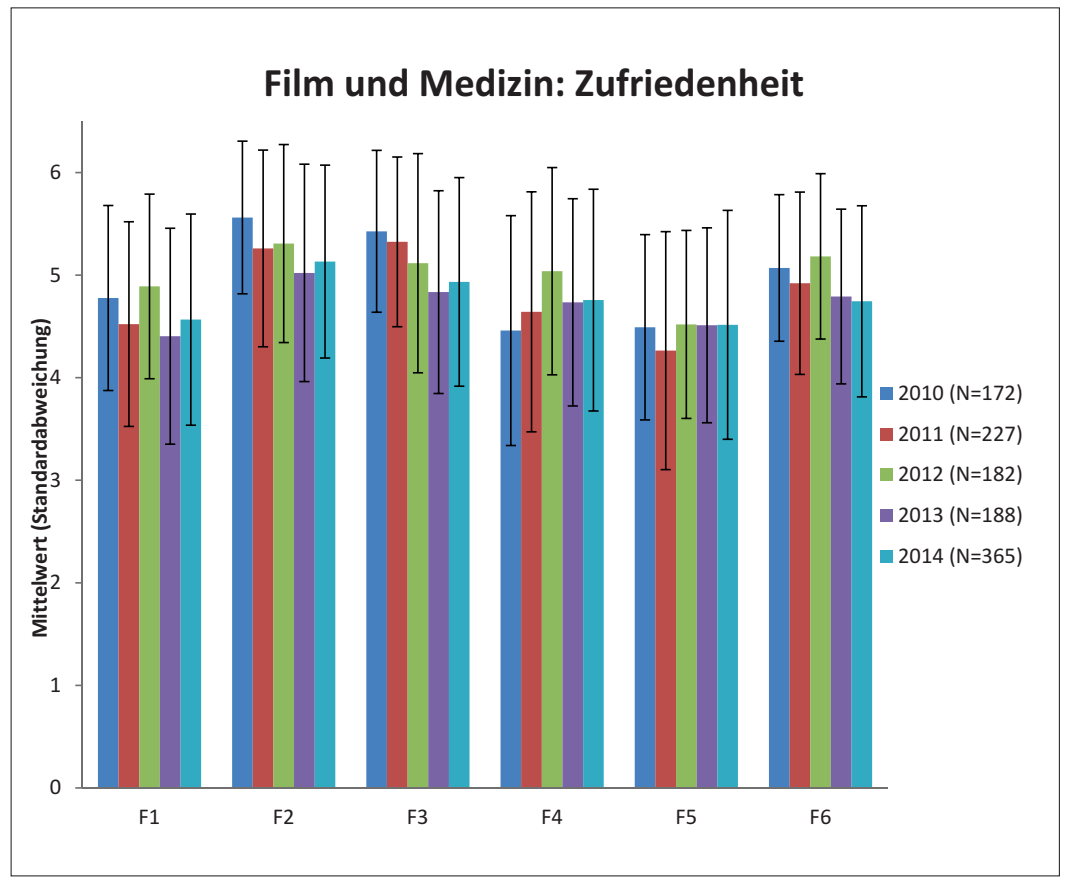

Abbildung 2: Zufriedenheit mit Film und Medizin. Die Studierenden antworteten mit einer 6-stufigen Skala: 1 = gar nicht zufrieden bis $6=$ sehr zufrieden. Zufrieden mit ... F1 der Einführung; F2 der Wahl des Films; F3 dem medizinischen Beitrag; F4 dem kulturwissenschaftlichen Beitrag; F5 den Diskussionen; F6 der Veranstaltung insgesamt.

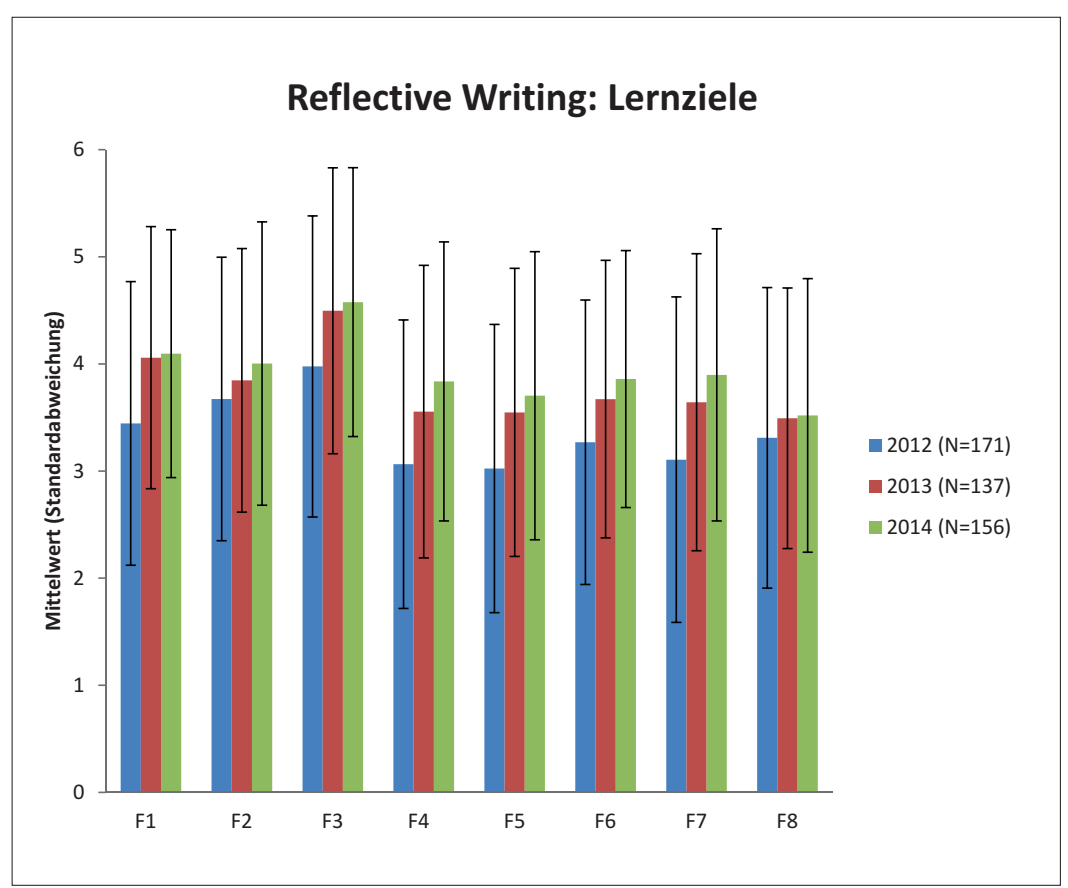

Abbildung 3: Lernziele Reflective Writing. Die Studierenden antworteten mit einer 6-stufigen Skala: 1 = trifft überhaupt nicht zu bis $6=$ trifft voll und ganz zu. Reflective Writing... F1 hat meinen Blick auf den Patienten geschärft; F2 hat mir geholfen, den Patienten genauer zu beschreiben; F3 hat mich dazu angeregt, über Patienten nachzudenken; F4 hat mir geholfen, meine Gedanken, Gefühle und Haltungen bestimmten Patienten gegenüber besser zu verstehen; F5 hat mir zu neuen Einsichten verholfen; F6 hat mir geholfen, für zukünftige Patientenkontakte konkrete Schlussfolgerungen zu ziehen; F7 ist ein geeignetes Mittel, um Patientenbegegnungen zu verarbeiten/reflektieren; F8 ist ein geeignetes Mittel, um die ärztliche Erzählkompetenz im Umgang mit Patienten zu schulen und zu verbessern. funden haben"). Das Feedback zu ihren klinischen Fähigkeiten sowie ihrem ausformulierten Text erhalten sie von ihrem jeweiligen Hausarzt und Tutor.

Da Lehrangebote in Medical Humanities bisher nur selten evaluiert wurden, war es für die Basler Projektgruppe interessant zu erfahren, wie zufrieden die Studierenden mit diesem Lehrangebot sind, ob aus ihrer Sicht die Lernziele erreicht werden und wie sich die Evaluation der Studierenden über die Zeit verändert. Kirkpatrick und Kirkpatrick [4] postulieren, dass Trainings bzw. Lehrveranstaltungen auf vier Ebenen evaluiert werden können: die Reaktion bzw. Zufriedenheit der Lernenden, der subjektive bzw. objektive Lernerfolg, das Verhalten in realen Situationen und Ergebnisse bzw. Outcomes. Entsprechend wurde mit Fragebögen zu «Film und Medizin» und "Literatur und Medizin» die Zufriedenheit mit Form und Inhalt sowie dem subjektiven Lernerfolg in Bezug auf die Lernziele der Veranstaltung erfragt (Abb. 2 und 3). Die jeweilige Antwort erfolgt auf einer 6-stufigen Skala ( $1=$ trifft überhaupt nicht zu bis $6=$ trifft voll und ganz zu). Die Evaluation des Reflective Writing orientierte sich an Reis et al. [5] und besteht aus 8 Fragen zu den Lernzielen.

Alle Lehrveranstaltungen der Medical Humanities wurden von den Studierenden unmittelbar am Ende der Veranstaltung oder bei Abgabe des Reflective Writing mit den oben genannten Fragebögen evaluiert. Die Studierenden wurden informiert, dass das Ausfüllen der Qualitätskontrolle diene und die Datenanalyse anonym durchgeführt werde.

Die Resultate, die einen Zeitraum von 5 Jahren umfassen, waren äusserst erfreulich. Beispielhaft sind in den Abbildungen die Ergebnisse des Blocks «Film und Medizin» dargestellt (Abb. 2). Hier wurden besonders die Auswahl des Films und die Beiträge der Kliniker gelobt. Aber auch das Ziel, bestimmte Themen (z.B. Sterben und Tod, Umgang mit Krankheit) anders als üblicherweise im Medizinstudium durch einen Film erlebbar zu machen, fand bei den Studierenden ausgesprochene Zustimmung. Eine Erhöhung der Besuchspflicht erklärt den deutlichen Anstieg der evaluierenden Studenten im Jahr 2014. Die Ausweitung der Anwesenheitspflicht hatte allerdings keinen Einfluss auf die Zufriedenheit der Studierenden. Vielmehr wurde der subjektive Lernerfolg im Jahr 2014 im Vergleich zum Vorjahr signifikant besser bewertet, was ebenfalls gegen einen negativen Einfluss der vermehrten Anwesenheitspflicht spricht.

Beim Themenkreis "Literatur und Medizin" finden sich übereinstimmende Resultate. Hier hatte die Ausweitung der Anwesenheitspflicht - im Vergleich zum Vorjahr - keinen Einfluss auf die Erreichung der Lern- 
ziele, dafür war die Zufriedenheit der Studierenden signifikant höher. Die Evaluation der Lernziele von Reflective Writing ist in Abbildung 3 ersichtlich. Offensichtlich ist es gelungen die Studierenden zum Nachdenken über ihre Patientenbegegnungen anzuregen. Der Lernerfolg dieser Massnahme hat sich über die Jahre kontinuierlich verbessert. Dies ist umso erfreulicher, als gerade diese Lehrveranstaltung eines beachtlichen Koordinationsaufwandes bedarf und von den beteiligten, Feedback gebenden Tutoren zusätzliches Engagement und nicht zuletzt ebenfalls Selbstreflexion fordert.

\section{Der mögliche Transfer von Medical Humanities in den späteren klinischen Alltag soll immer aufgezeigt werden.}

Unsere Resultate zeigen, dass Studierende Medical Humanities als Pflichtteil ihres Curriculums akzeptieren und aus ihrer Sicht die Lernziele überwiegend erreicht werden. Die vermehrte Präsenzpflicht bei den Veranstaltungen hatte keinen negativen Einfluss auf die Zufriedenheit und den subjektiven Lernerfolg der Studierenden. Erfreulicherweise ist es auch bei diesen sehr individuell gestalteten Veranstaltungen möglich, über längere Zeit ein vergleichbares Niveau zu halten.

Uns ist in der wissenschaftlichen Literatur keine Arbeit bekannt, in der ein obligates Medical Humanities Curriculum für Studierende über mehrere Jahre evaluiert wurde. Kritisch anzumerken ist, dass sich die beschriebene Evaluation auf die Einstellungen der Studierenden beschränkt: Wenn Studierende im Fragebogen angeben, dass sie durch Reflective Writing Patienten besser verstehen und konkrete Schlussfolgerungen für zukünftige Patientenkontakte gezogen haben, heisst dies nicht zwingend, dass diese Einschätzung in der realen Situation auch wirklich zum Tragen kommt und darauf Einfluss hat, wie sie tatsächlich mit Patienten umgehen. Selbsteinschätzung korreliert häufig nicht mit Verhalten. Um jedoch messen zu können, inwieweit der Einbezug von Medical Humanities ins Medizinstudium zu Verhaltensänderungen oder sogar einer verbesserten Patientenversorgung führen könnte, ist es notwendig, neue Evaluationsinstrumente zu entwickeln.

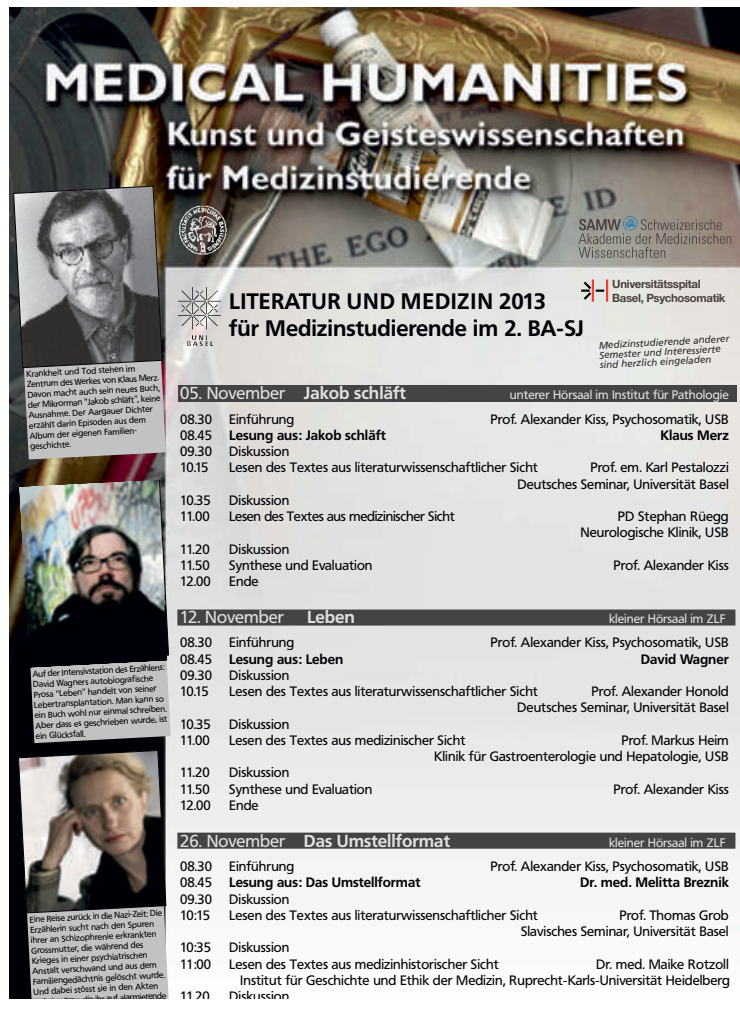

Abbildung 4: Programmankündigung der Veranstaltungen zu "Literatur und Medizin».

Beschränkt auf Lehrveranstaltungen von Medical Humanities ist die studentische Evaluation ein brauchbares Mittel um die Akzeptanz bei Studierenden zu verbessern, eine gewisse Qualitätskontrolle der Lehre zu sichern und die Weiterentwicklungen von Medical Humanities im Curriculum zu ermöglichen.

\section{Literatur}

1 Akademien der Wissenschaften Schweiz (Hg.). Medical Humanities in der Schweiz. 2012 (www.akademien-schweiz.ch/ $\mathrm{dms} / \mathrm{D} /$ Publikationen/Berichte/MedicalHumanities_d.pdf).

2 Louis-Courvoisier M, Wenger, A. How to make the most of history and literature in the teaching of medical humanities: the experience of the University of Geneva. J Med Ethics. Medical Humanities. 2015;31:51-4.

3 Isler R, Romerio S, Halter, U, Heiniger S, Persike M, Roers, B et al. One-on-one long-term tutorials in general practitioners' practices - a successful new teaching concept in primary care medicine. Swiss Med Wkly. 2009;139(11-12):161-5.

4 Kirkpatrick DL, Kirkpatrick JD. Evaluating Training Programs. The four levels. San Francisco: Mcgraw-Hill Professional; 2006

5 Reis SP, Wald HS, Monroe AD, Borkan JM. Begin the BEGAN (The Brown Educational Guide to the Analysis of Narrative) a framework for enhancing educational impact of faculty feedback to students' reflective writing. Patient Educ Couns. $2010 ; 80(2): 253-9$. 